\title{
Uterine Arterio-venous Malformation with Hemoperitoneum
}

\section{Rajshree D. Katke', Shilpa Domkundwar' ${ }^{2}$, Sunil C. Rathod'}

From the Department of Obstetrics \& Gynecology ${ }^{1}$ and Department of Radiology ${ }^{2}$, Cama

\& Albless Hospital, Grant Government Medical College \& Sir J. J. Group of Hospitals,

Mumbai, Maharashtra, India.

Abstract:

Arterio-venous malformations (AVM) are rare entities in gynecology, with only over 100 cases reported since 1926. These lesions may be congenital or acquired. Most commonly they present with vaginal hemorrhage, but other presentations such as congestive heart failure, postmenopausal bleeding and an asymptomatic mass have been described. We report a 28 year old female with endometrial polyp and AV malformation with massive hemo-peritoneum. Uterine A-V malformations are rare cause of menorrhagia, but they should always be kept in mind in patients with previous operative history on uterus.

Key words: Arteriovenous malformations, Uterine hemorrhage, Heart failure, Menorrhagia, Polyps.

Introduction

Congenital uterine $A V M s$ are thought to develop secondary to faulty arrest in the angiogenic process [1,2]. Acquired AVMs are thought to be related to uterine trauma including curettage or cesarean delivery, retained products of conception, chorio-carcinoma, endometrial or cervical carcinoma, gestational trophoblastic disease and diethylstilbestrol exposure. Most commonly they present with vaginal hemorrhage, but other presentations such as congestive heart failure, postmenopausal bleeding, and an asymptomatic mass have been described [1-3]. Diagnosis can rapidly be made with color flow Doppler ultrasound or angiography as in our reported case.
Case Report

28 year Para-1, Living-1, Abortion-1 married since 5 years, HIV positive female presented with abdominal pain, bleeding per vaginum with passage of clots since last 24 hours. Her past history was insignificant except previous history of dilation and currettage one and half year back. Her outside ultrasound gave differential diagnosis of ruptured ectopic, ruptured hemorrhagic cyst, vascular mass within endometrial cavity or polyp. When patient came to our institution she was in a state of haemorrhagic shock with pulse rate of 112 beats/min, blood pressure of 94/62 $\mathrm{mmHg}$. Per abdomen examination revealed distension of lower part of abdomen, severe tenderness and guarding

Corresponding Author: Dr. Rajshree D. Katke

Email: drrajshrikatke@gmail.com

Received: December 19, 2013 | Accepted: January 22, 2014 | Published Online: February 10, 2014 This is an Open Access article distributed under the terms of the Creative Commons Attribution License (creativecommons.org/licenses/by/3.0)

Conflict of interest: None declared | Source of funding: Nil | DOl: http://dx.doi.org/10.17659/01.2014.0015 
in lower abdomen with clinically no mass felt. Her speculum examination was suggestive of mild fresh bleeding while per vaginal examination revealed bulky tender uterus. Cervical movements were tender and forniceal tenderness was present. Investigations showed negative urine pregnancy test,", hemoglobin of $7.6 \mathrm{gm} / \mathrm{dL}$, beta HCG $13 \mathrm{IU} / \mathrm{mL}$, with normal liver and renal function tests. We kept a provisional diagnosis of ruptured hemorrhagic cyst. Her urgent Doppler ultrasound was suggestive of bulky uterus with relatively well defined heterogenous predominantly hyperechoic lesion giving whorled appearance. The $4.2 \times 4.4 \times 3.5 \mathrm{~cm}$ mass protruding into the endometrial cavity showed cystic anechoic space within showing mixed AV waveform with aliasing of color. The arterial supply appeared to be directly arising from the left uterine artery with machinery murmur and arterialization of left uterine venous waveform with evidence of active ongoing bleed. Moderate to gross free fluid in abdomen with internal echoes were suggestive of hemo-peritoneum [Fig. 1,2].

Our impression was uterine artery AV malformation with surrounding heterogenous hyperechoic lesion occurring secondary to endometrial polyp or organized hemorrhage with active bleed. However, the size of hemo-peritoneum was disproportionate to the size of AVM and so the exact etiology of

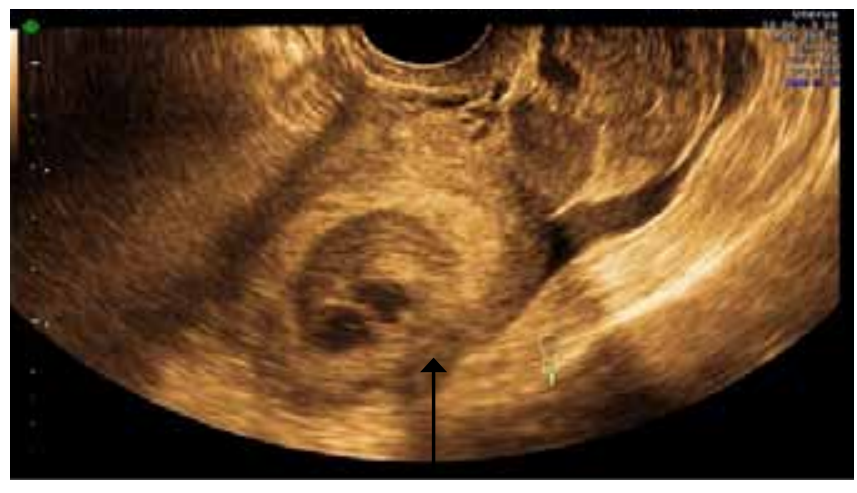

Fig.1: Ultrasound showing A-V malformation in endometrial cavity. hemo-peritoneum could not be determined.

As patient was in state of hemorrhagic shock with hemo-peritonium and keeping in mind the dilemma in regards to diagnosis of the patient and decision of emergency exploratory laparotomy was taken and if required then hysterectomy. Intraoperatively, uterus was bulky and blood was pouring through both tubal ostia suggestive of bleeding from the endometrial cavity. Both the fallopian tubes and ovaries were normal. As the bleeding was from endometrial cavity which had resulted in hemoperitoneum; the decision of hysterectomy was taken. Total abdominal hysterectomy was performed and 1.5 liters of hemorrhagic fluid was drained. Four pints of blood and 2 pints of fresh frozen plasma were transfused. On cutting the specimen of uterus a vascular polyp approximately of $4.5 \times 4 \times 5 \mathrm{~cm}$ was seen arising from uterine fundus with 100 grams blood clot filling the entire endometrial cavity just below the polyp [Fig.3]. Patient tolerated surgical procedure well. Her post-operative period was uneventful.

\section{Discussion}

Regardless of the etiology, uterine AVMs are a potential source of significant morbidity and,

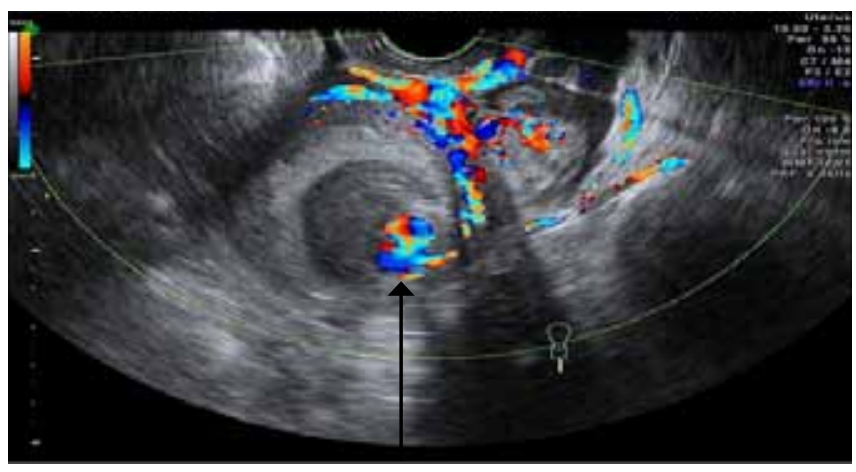

Fig.2: Doppler ultrasound shows mixed AV waveform with aliasing of colour suggestive of A-V malformation with in endometrial cavity. 
rarely, mortality. The true incidence is unknown, but the majority of cases are found in women of reproductive age. Most commonly, they present with menorrhagia or meno-metrorrhagia requiring blood transfusions in 30\% of reported cases [3]. In our case, the patient presented with hemoperitoneum and state of hemorrhagic shock as unusual presentation. The differentiation between a uterine $A V M$ and other causes of uterine bleeding is necessary because instrumentation can lead to massive hemorrhage.

Historically, uterine AVMs were diagnosed by laparotomy or pathologically after hysterectomy. Angiography is the gold standard for diagnosis, classically showing complex vascular connections supplied by hypertrophied feeding arteries and early drainage through enlarged and hypertrophic veins. Although angiography has the ability to assess the size of AVMs and vessel involvement, it is invasive and cannot accurately detect the degree of pelvic extension. Beside it needs expertise and the cath lab is not available everywhere. Ultrasound and MRI accurately delineate the extent of adjacent organ involvement and are now considered the modalities of choice when evaluating a patient with suspected uterine AVM. Again the cost of MRI and its availability are prohibitive. Ultrasound with Doppler study remains the modality of choice in day to day practical life. As this patient presented with hemo-peritoneum leading to hemorrhagic shock, exploratory laparotomy was done as a life-saving procedure.

Gray-scale ultrasound features of uterine AVMs include multiple anechoic structures with a serpentine contour within the myometrium [4]. However, these features are often confusing and nonspecific $[3,4]$. The addition of color and spectral analysis can lead to more accurate diagnoses. On color Doppler, these hypoechoic structures exhibit vascular flow. Huang et al suggested two distinct color patterns consistently associated with uterine

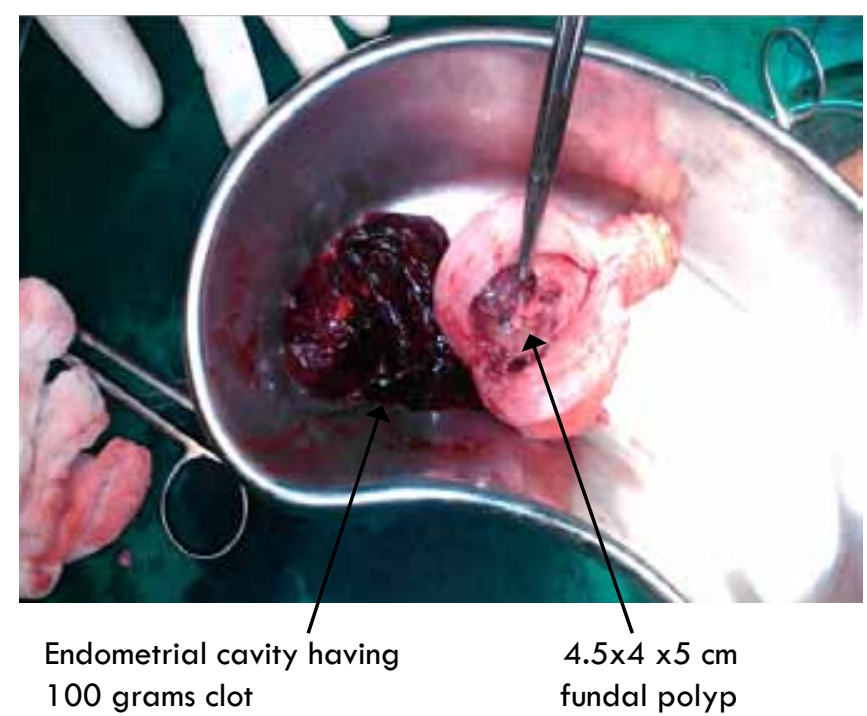

Fig. 3: Cut specimen of uterus.

AVMs. The first is the juxtaposition of blue and red components, suggesting blood flow reversals caused by overlapping vessels of varying orientations and flow directions. The second is color aliasing and the separation of red and blue components by yellow and white, suggesting focal high velocities [3]. Spectral Doppler demonstrates low-resistance and high-peak systolic velocities with continuous high flow throughout systole and diastole $[2,3]$.

MRI provides accurate definition of uterine AVMs and effectively delineates invasion of adjacent organs. Characteristic features include a bulky uterus with a focal mass, disruption of the junctional zones, multiple serpiginous flow-related signal voids within the lesion, and prominent parametrial vessels $[2,3]$. Gadolinium-enhanced MRI demonstrates hypervascular arterial dominant flow [5-7]. But it can be used only in stable patients and only a few affluent patients can be advised MRI.

Prior to embolotherapy, hysterectomy or uni/ bilateral uterine artery ligation were the therapies of choice. Since the first reported case of transcatheter uterine artery embolization for a uterine AV fistula in 1982, embolo-therapy has become a well- 
recognized alternative to surgical intervention for uterine AVMs, with the major advantage of maintaining childbearing capacity. Ghai et al recently reported a retrospective review of all patients who underwent pelvic arterial embolization for traumatic uterine $A V M s$ at their institution during a 10-year span. The authors concluded that selective uterine artery embolization is a safe and effective treatment for traumatic uterine AVMs while preserving the possibility of future pregnancy [8]. Gelfoam offers 3 to 5 weeks of occlusion that is sufficient to occlude the AVM while still permitting slow development of collateral vessels, allowing for the preservation of fertility and resumption of menstruation. Furthermore, an increasing number of pregnancies following transcatheter embolization of uterine AVMs are being reported $[8,9]$.

The current literature, suggests that transcatheter uterine artery embolization of traumatic uterine AVMs is a safe and effective alternative to surgical therapy while offering the major advantage of preserving childbearing capacity. But in our case, patient had abnormal presentation i.e. hemoperitoneum so emergency exploratory laparotomy with total abdominal hysterectomywas performed.

\section{Conclusion}

Uterine $A-V$ malformation are rare cause of menorrhagia, and ultrasound with Doppler study remains the modality of choice in day to day practical life.

\section{References}

1. Grivell RM, Reid KM, Mellor A. Uterine arteriovenous malformations: a review of the current literature. Obstet Gynecol Surv. 2005;60:761-767.

2. Huang MW, Muradali $D$, Thurston WA. Uterine arteriovenous malformations: gray-scale and Doppler US features with MR imaging correlation. Radiology. 1998;206:1 15-123.

3. Hoffman MK, Meilstrup JW, Shackelford DP, Kaminski PF. Arteriovenous malformations of the uterus: an uncommon cause of vaginal bleeding. Obstet Gynecol Surv. 1997;52:736-740.

4. Bagga R, Verma P, Agarwal N, Suri V, Bapurai $J R$. Failed angiographic embolization in uterine arterio venous malformation: a case report and review of literature. Available at www. medscape.com/viewarticle /567523_print. Accessed on 15 December 2013.

5. Nicholson AA, Turnbull LW, Coady AM, Guthrie $K$. Diagnosis and management of utrine arteriovenous malformations. Clin Radiol. 1999;54(4)265-269.

6. Nasu K, Fujisawa K, Yoshimatsu J, Miyakawa I. Uterine arteriovenous malformation: ultrasonographic, magnetic resonance and radiological findings. Gynecol Obstet Invest. 2002;53:191-194.

7. Gupta S, Geetika, Bansal R, Chauhan H. Uterine Leiomyosarcoma: a Management Dilemma. Journal of Case Reports. 2013;3:134-136.

8. Kwon JH, Kim GS. Obstetric iatrogenic arterial injuries of the uterus: diagnosis with US and treatment with transcatheter arterial embolization. Radiographics. 2002;22:35-46.

9. Ghai S, Rajan DK, Asch MR. Efficacy of embolization in traumatic uterine vascular malformations. J Vasc Interv Radiol. 2003;1 4:1401-1408. 